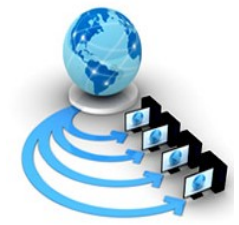

Volume 9, No. 5, September-October 2018

\title{
ENERGY EFFICIENT CLUSTERING TECHNIQUE USING LEACH PROTOCOL FOR WIRELESS SENSOR NETWORK
}

\author{
Surbhi Saraf, \\ Computer Science\& Engineering, \\ Adina Institute of Science\& Technolog, RGPV, Sagar, India
}

\author{
Rajneesh Pachouri \\ Computer Science\& Engineering, \\ Adina Institute of Science\& Technolog, RGPV, Sagar, India
}

\author{
Anurag Jain \\ Computer Science\& Engineering, \\ Adina Institute of Science\& Technolog, RGPV, Sagar, India
}

\begin{abstract}
Sensor Network helps in watching distinctive sorts of condition by distinguishing the physical atmosphere. Our noteworthy focus lies on extending the framework lifetime with the objective that the battery require not to be recharged after a short time. cluster sensor nodes is a feasible technique for achieving this target. In this work, we present an essentialness compelling Clustering estimation for sensor frameworks subject to the LEACH Protocol. LEACH (Low Energy Adaptive Clustering Hierarchy) is one of conspicuous group based structures, which has been by and large proposed in wireless sensor Networks. The proposed protocol LEACH-E (Improved LEACH protocol) has bolstered office that it diminishes the use of the framework resource as stand out from LEACH figuring in each round. The proposed tradition is re-sanctioned and the result shows a colossal abatement in framework essentialness use when stood out from LEACH. Our genuine spotlight relies upon boosting Nodes degree that is portrayed as the amount of alive center points that exist in the transmission extent of a given center is more as appear differently in relation to LEACH estimation.
\end{abstract}

Keywords - WSN, CH, LEACH, E-LEACH， ENERGY

\section{INTRODUCTION}

As of late, scientists have been pulled in by wireless sensor network (WSN) because of their potential use in a wide assortment of utilizations. A WSN contains distinctive sorts of self-governing sensor nodes that are utilized to detect and exchange the information remotely to the Base Station (BS) or the following recipient node. Ordinarily hundreds or thousands of minimal effort sensors are utilized in Wireless sensor network [1]. Directing is an issue for these sensor nodes where the re-sources are restricted. These remote sensor nodes have restricted vitality, preparing capacities, and detecting capacities. At first WSNs were utilized just in the front lines for military purposes however now their utilization is reached out for observing and controlling the distinctive procedures in numerous other regular citizen territories. An extensive variety of sensors are accessible to screen the distinctive surrounding conditions, for example, temperature, weight, moistness, development, and helping conditions [2]. The detected data and totaled information conveyance is fundamental for productive correspondence between sensor nodes. In a social insurance framework, the embed and wearable sensors associated with a human body send the information to the facilitator node, which exchange these information towards the correspondence level 2 gadgets. The level 2 gadgets at that point course these information to the focal database server for further preparing [3].

The data is steered from the root node to the Base station either straightforwardly or through other sensor nodes. The BS is either a settled or a versatile node which is fit for interfacing the system to the web where client can access and process the information. Directing in WSN is extremely testing because of the natural attributes that recognize this system from different remote systems or cell systems. The most vital imperative on WSN is the constrained battery power or sensor nodes. The required lower vitality utilization confines the sensor to utilize the constrained assets, for example, less memory limit, low transmit control, and less handling calculations.

\section{LEACH PROTOCOL}

Low energy adaptive clustering hierarchy system is various leveled convention which enables the nodes to transmit information to the group heads of the cluster to which they have a place. The cluster heads total the information got from the non-group head hubs or group part and forward it to the Base Station (Sink). It is a cluster based steering convention whose fundamental point is to build the lifetime of the wireless sensor network. It is a decent case of self-versatile and selfsorted out convention. Its general task depends on rounds and each round comprises of two phases setup stage and steady_state stage.

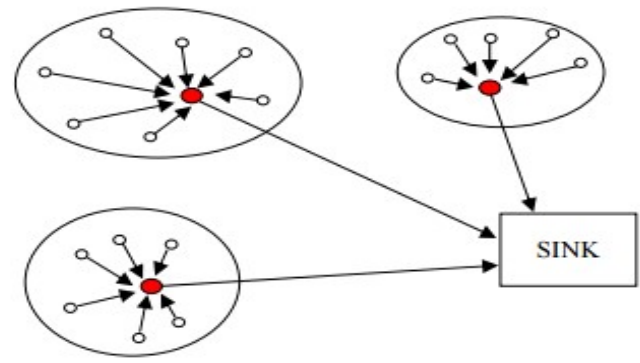

Fig1.. Architecture of LEACH Protocol 
The actual thought of LEACH protocol lies in separating the entire network into different clusters. In each cluster, a group head is chosen in various leveled way and this job is pivoted among the nodes in the cluster with the end goal to homogeneously convey the power stack in each round. This protocol permits versatility and power in the system and likewise helps in packing the measure of data to be sent to the Base Station. The fundamental design is delineated in figure 1.

leach Protocol is a well delegate of various leveled directing conventions. It is self-versatile and selfcomposed. Filter convention utilizes round as unit, each round is comprised of group set-up stage and consistent state organize, to reduce superfluous vitality costs, the unfaltering state arrange must be any longer than the setup stage.

\section{HOMOGENEOUS CLUSTERING PROTOCOLS}

\section{a) $\mathrm{LEACH}$}

Drain is characterized as low vitality versatile bunching progressive system. In this convention a head is picked among an arrangement of hub haphazardly and that hub is then called to be group head $(\mathrm{CH})$. On the off chance that every hub sends the information to base station then most extreme of system vitality gets squandered for transmission so to beat this overhead a hub is picked as a head and all other hub sends their information to head $(\mathrm{CH})$ and this diminishes the vitality heap of the system. Barely any primary component of LEACH are pressure of information before sending it to sink hub, arbitrary determination of group head and most vital is to keep up a co-appointment between non $\mathrm{CH}$ hubs and $\mathrm{CH}$ hub.

$\mathrm{CH}$ is decided to the hub which great than other hub in each perspective in light of the fact that $\mathrm{CH}$ is the hub which needs to speak with all other typical hubs in the bunch which are put far off in the group. Drain is a convention which changes the $\mathrm{CH}$ occasionally on the grounds that vitality of bunch head turns out to be low while speaking with different hubs after one round so trade of $\mathrm{CH}$ is improved the situation the following round. Undertaking of $\mathrm{CH}$ is to pack the information gathered by ordinary hubs of a similar group and send that total information to the sink hub or base station. Information gathering is brought together and intermittently. Anyway the issue is information isn't required intermittently and a considerable measure of vitality is squandered because of pointless information. Filter performs in two stages 1) Setup state and 2) unfaltering stage.

Setup stage: Selection of bunch head which is arbitrary if there should arise an occurrence of LEACH is under the setup stage. A foreordained part of hubs i.e. $p$ is expected to choose $\mathrm{CH}$ among themselves as it were. Every hub in bunch chooses an irregular number somewhere in the range of 0 and 1 and a limit a chose i.e. T(n). On the off chance that arbitrary number is not as much as a $T(n)$ at that point the hub turn into the group head forward at round. But limit a hub must fulfill one more condition to end up group head. The condition is that hub was not chosen as $\mathrm{CH}$ in last (1/p) rounds. On the off chance that a hub fulfills both the conditions then the likelihood of getting to be $\mathrm{CH}$ for that hub turn out to be high. After determination of $\mathrm{CHs}$ for each bunch, $\mathrm{CHs}$ communicate commercial to all non group head hubs $\mathrm{CH}$ utilizes CSMA convention to communicate the notice message to all hubs in a similar group and dependent on the flag quality typical hub joins the bunch and send an affirmation message to $\mathrm{CH}$. At the point when all hubs do a similar then $\mathrm{CH}$ makes a TDMA plan and apportions a period quant to each hub. Hubs must transmit the information in the allocated time quant. This timetable must communicate to each hub.

Consistent stage: In this stage all hubs transmits the information and $\mathrm{CH}$ totals the gathered information and afterward send it to base station after that round gets completed and after that again for the following round setup and enduring state begins. Each Cluster utilizes diverse CDMA codes with the end goal to decrease the impedance between them.

After investigation of homogeneous conventions it is being examined that LEACH is most well known among specialists and from 10 years analysts endeavors to enhance it by including distinctive parts of other region like including differential development from arithmetic, subterranean insect province advancement from swarm insight and some more. Also, it is apparent that there are in excess of 20 assortments are as of now proposed and they were talked about quickly in next segments.

\section{b) ALEACH}

ALEACH is characterized as Advance LEACH. In this LEACH bunch development is somewhat not the same as the fundamental LEACH; Clusters were framed dependent on selfsufficient choice of hubs in the system with no obstruction of focal control. Bunches were shaped without knowing the correct area of different hubs of the group and no worldwide correspondences is expected to frame a group. In this convention what makes a difference is the present condition of the hub. Choice of bunch head is very unique in A LEACH. In fundamental LEACH $\mathrm{CH}$ is picked dependent on the edge esteem, T(n) which relies upon the round without knowing the condition of different hubs of the group yet in ALEACH this isn't the situation. In ALEACH choice of group head isn't just relies upon round yet in addition on the present vitality. On the off chance that two hubs have diverse current vitality then the hub having higher current vitality is picked as $\mathrm{CH}$, with the goal that all beyond words a similar time, this is the real thought behind that run the show.

So in the wake of considering the present state vitality the limit condition moves toward becoming

Subsequent to having bunch set out toward the round, $\mathrm{CH}$ makes a TDMA calendar and transmits to the group with the goal that it is known to every one of its individuals. Presently the hubs send the information to $\mathrm{CH}$ following the TDMA timetable to keep away from the impact amid the transmission amid the enduring state stage.

\section{c) MR-LEACH}

Multi-jump steering LEACH isolates the system into layers of bunches and for each group, $\mathrm{CH}$ is picked. $\mathrm{CH}$ of highest level (Chosen by Base station) goes about as director for them and guides them to course the information towards the base station. CHs in MR-LEACH go about as gathering hub as well as go about as transferring hubs to course the information to the base station. Before disclosure of MR-LEACH, a few conventions were found which pursues the multi-bounce directing with unequal dividing of groups. 
This sort of apportioning makes an issue in planning in view of unequal groups. To take care of this issue CSMA (bearer sense various access) is utilized which is very costly in examination with TDMA. So equivalent bunches rises which lessens the normal separation between each group which indicates change in outstanding vitality consequently increment the system lifetime. Since number of bunches was equivalent so they can utilize TDMA plan.

\section{d) Balanced LEACH}

Determination of $\mathrm{CH}$ depends on the leftover vitality of the hubs. On the off chance that the quantity of $\mathrm{CH}$ in the system is not as much as $n^{*} \mathrm{p}$ then a clock is made and as of now interim a notice for $\mathrm{CH}$ is send among the hubs of the bunch yet on the off chance that the clock lapses at that point change to $\mathrm{CH}$ and again communicate for $\mathrm{CH}$ ad. On the off chance that the quantity of $\mathrm{CH}$ isn't not as much as $n^{*} \mathrm{p}$ at that point arranging of $\mathrm{CH}$ is done dependent on the remaining vitality and the $\mathrm{CHs}$ which have leftover vitality not as much as normal vitality must kill from the $\mathrm{CHs}$ list.

\section{e) Re-group LEACH}

Basic- LEACH convention is a solitary bounce convention and it doesn't think about the rest of the vitality of the hub. It just chooses the $\mathrm{CH}$ from entire system in each round, while in re-group LEACH CH is chosen inside a similar bunch for each round. In any case, the principle contrast among LEACH and re-group LEACH is the system to make the bunches. Re-bunch LEACH frames the groups dependent on the hub thickness in a zone. After arrangement of group and determination of $\mathrm{CH}$, information transmission is done dependent on CSMA/CD rather than TDMA.

\section{f) LEACH-C}

In concentrated LEACH subsequent to bunching, every hub sends its remaining vitality and additionally their ID to the construct station and based with respect to the higher lingering vitality base station chooses the $\mathrm{CH}$. This the significant contrast between essential LEACH and LEACH-C , $\mathrm{CH}$ determination isn't inside the group yet outside the bunch by the base station. Henceforth base station knows about each hub in the system and in the wake of picking the $\mathrm{CH}$, base station communicate the ID of $\mathrm{CH}$, so those individuals from group think about their $\mathrm{CH}$. It utilizes GPS system to get the area of hubs however it isn't hearty.

\section{g) Energy LEACH}

It pursues the enhanced determination of bunch head Residual vitality is the choice factor in this calculation. In this calculation, $\mathrm{CH}$ is chosen arbitrarily for the first round on the grounds that around then leftover vitality is same for every hub and after first round every hub have diverse lingering vitality. Based on remaining vitality, choice of group head has been improved the situation each round after first round. After choice of $\mathrm{CH}$, a TDMA plan shapes for information transmission in relentless state stage.

\section{h) Fixed number of bunch LEACH}

Drain F is especially like LEACH-C. Base station creates the bunch head arrange and dependent on this $\mathrm{CH}$ job is pivoted between the hubs. After the grouping calculation, the $\mathrm{CH}$ job arrangement begins. Preferred standpoint of LEACH-F over other $\mathrm{LEACH}$, there is no overhead of outlining a calculation for bunch head determination and it nearly less expensive as no $\mathrm{CH}$ choice for each round. Be that as it may, it doesn't take care of the issue of getting to be vitality productive convention.

\section{i) TL-LEACH (Two Level-LEACH)}

Two-level LEACH convention hubs partitions themselves into bunch self-sufficiently without intercession of focal control and grouping depends on hub thickness. To spare the vitality utilization the hubs are isolated into progressive system of bunches, so message from the most extreme of hubs doesn't have to venture to every part of the bigger separation. The system has top bunch head then auxiliary group head and afterward straightforward hubs at the most reduced level. It pursues the CSMA plot for planning.

\section{j) V-LEACH}

VLEACH remains for bad habit bunch head LEACH. Rather than having $\mathrm{CH}$ for information transmission, there is bad habit $\mathrm{CH}$ moreover. At the point when the heap on $\mathrm{CH}$ builds then the leftover vitality of that hub diminishes and soon it winds up kicking the bucket. All things considered the information gathered amid a round will squander in view of death of $\mathrm{CH}$. To take care of this issue a hub under the $\mathrm{CH}$ is allocated who will work after the $\mathrm{CH}$ passing with the goal that gathered information won't lost and reach to the base station. It additionally enhances the system lifetime.

\section{k) LEACH-M}

This convention had a slight change over fundamental LEACH is that relentless state stage bolsters the portability of hubs. TDMA plan is amended each time when another hub touches base at the group. At the point when a hub sends an information and the information doesn't reach to the $\mathrm{CH}$ then it expect that hub must go into some other group and vacancy for that hub must be expelled from the $\mathrm{CH}$ memory, and $\mathrm{CH}$ of close-by bunch adds that hub to its very own bunch and refresh its TDMA plan. To get the area data of the hub GPS system is utilized.

\section{1) VR-LEACH}

Variable round LEACH, as the name propose, round for the information transmission must be variable long. In the setup stage, those hubs were killed which have vitality not as much as the normal vitality of the system. Provided that the hub with vitality not as much as normal vitality chooses as $\mathrm{CH}$ then it will bite the dust soon and the information accumulated by them will lost. Rest of the hub took an interest in the $\mathrm{CH}$ race. After that most suitable hub is chosen as $\mathrm{CH}$ and bunch development begins then $\mathrm{CH}$ makes and communicate the TDMA calendar to each hub of the group and information transmission starts. Contingent upon the length of round time, a next round starts. Round time changes if a portion of the hub will bite the dust from the group and won't ready to take part for information transmission at that point space involved by them must expel and change the round length, so LEACH works legitimately and effectively.

\section{m) N-LEACH (Number of hubs upheld in past round)}

On the off chance that number of hubs inside a similar group head expands then the heap on that hub likewise increments. As the LEACH pursues the plan to join the bunch dependent on the separation from the $\mathrm{CH}$. Hubs joins the $\mathrm{CH}$ 
which was closest to them and along these lines hubs in the each bunch wind up uneven and stack on $\mathrm{CH}$ turn out to be likewise uneven. In NLEACH another technique for $\mathrm{CH}$ choice is presented which adjusts the vitality utilization of each hub and furthermore adjusts the heap. In this plan at first information transmission for all hubs move toward becoming $(-1)$ and check if hubs vitality is more prominent than zero at that point if the information transmission is more noteworthy than 0 and furthermore fulfills limit, Tn then the hub move toward becoming bunch head and information transmission increments by $(\mathrm{n} / \mathrm{k}) * \mathrm{~N}$. generally no determination of group head and next round begins.

\section{n) DE-LEACH (Differential development calculation LEACH)}

Untimely of death is the fundamental issue essential LEACH was confronting and scientists endeavor to tackle the issue by enhancing the LEACH calculation. Another calculation was proposed which utilizes Differential development calculation for bunch head choice. DE is utilized to enhance the multi-objective $\mathrm{CH}$ determination calculation and based on swarm the ID's of hub is arranged subsequent to completing the original and after that hybrid is held among them and after that choice happens dependent on adjustment. At that point group head is chosen in the wake of achieving the end condition. Presently DE-LEACH pursues indistinguishable track from the LEACH pursues. Be that as it may, this change in determination of $\mathrm{CH}$ is a checked history.

o) LEACH-ID (Identification based LEACH)

In this convention, a one of a kind ID is allocated with parallel number to every sensor hub. In light of this ID information is send utilizing a solitary way i.e. unicast rather than communicate which enhances the system lifetime and shows high vitality proficiency. These special ID's are brief and change after each round.

\section{II.RELATED WORK}

The past work in field of clustering is specified in this area. These work are additionally proficient and gives data about the work is as of now done in field of energy effeciancy.

Wang et al (2009) proposed a refined convention named LEACH-H (half breed group head determination LEACH) with the end goal to drag out the WSN's lifetime. In the initial round of $\mathrm{LEACH}-\mathrm{H}$, the base-station picked a $\mathrm{CH}$ set through getting Simulated Annealing (SA) computation; in the rounds, the $46 \mathrm{CHs}$ along these lines would pick new $\mathrm{CHs}$ in their very own gathering. This won't just take care of the issue that the CHs were unevenly disseminated in $\mathrm{LEACH}$, yet in addition keep up the attributes of appropriation. The vitality utilization of the system is chopped down and the live time of WSN is reached out in LEACH-H [4].

Y. He, Y. Zhang et al (2010) proposed to handle the strict constraint of vitality supplication in vast scale progressive structure of WSN. The proposed choice methodology in $\mathrm{CH}$ choice system thinks about distinguished flag quality, a hub's remaining vitality and separation between group head and sink hub. A versatile strategy dependent on assignment necessity in bunch part determination was proposed in regards to group run setting. This technique obliges group size and vitality utilization of intra-bunch correspondence is lessened.
Recreation demonstrated that the new convention saved vitality and delayed WSN life uncommonly when sink hub is a long way from the system. Considering the character of correspondence extent of hub in bunch, there exists zones of excess place between joined groups. To give an exact number of $\mathrm{CH}$, proficient inclusion is utilized, rather than full inclusion of group to compute the ideal number of $\mathrm{CH}$ per round, as per characters of one and two request fractional subsidiary capacities [5].

Yoon and Chang (2011) proposed another group based steering convention utilizing message achievement rate. To determine the hub fixation issue and plan another $\mathrm{CH}$ choice calculation dependent on hub network and devise bunch upkeep calculations. Besides, to ensure unwavering quality of information correspondence, message achievement rate is a prevalent measure for information correspondence dependability, is utilized with the end goal to choose a directing way. At long last, to decrease information correspondence overhead, just data of neighboring hubs amid both bunch head determination and group development stages were utilized. Through the execution investigation, it demonstrated that proposed convention outflanked the current plans as far as correspondence unwavering quality and vitality productivity $[6]$.

Peng et al (2011) was proposed A LEACH calculation based vitality compelling directing convention to meet key QoS prerequisites. Vitality productivity is a critical WSN issue. System layer directing innovation is basic to decrease WSN vitality utilization. Be that as it may, dependability and information hostility should likewise be investigated. The new convention concentrated on customary LEACH absconds and enhanced vitality effectiveness and QoS parameters by barring hubs with ill-advised geographic area to be CHs. Ideal estimating scope of head hubs is intended to be a $\mathrm{CH}$ determination paradigm and each $\mathrm{CHs}$ can be chosen by hub thickness limit in estimating region, affirmed by hub dispersion circumstance and correspondence needs. Recreations assessed the new convention in correlation with customary LEACH calculation. The execution of new convention was confirmed to diminish vitality utilization and certification correspondence quality particularly in uneven conveyance situations[7].

Kuila and Jana (2012) introduced a circulated grouping and steering calculation for WSN called Cost-based Energy Balanced Clustering and Routing Algorithm (CEBCRA). The calculation contained three stages, specifically $\mathrm{CH}$ choice, bunch setup and information steering. The $\mathrm{CHs}$ were chosen in a dispersed way dependent on remaining vitality and the neighbor cardinality. In the setup stage, each non- $\mathrm{CH}$ sensor hub joined a $\mathrm{CH}$ inside its correspondence go dependent on the cost estimation of the CHs. In information steering stage, CEBCRA originally utilized single-jump correspondence inside each group and afterward performed multi-bounce correspondence between the bunches. For inter cluster directing, a $\mathrm{CH}$ estimated the expense of every way from itself towards base station while choosing other $\mathrm{CH}$ as a transfer hub for information sending on those ways. The test results demonstrated productivity of the proposed calculation regarding vitality utilization and number of live sensor hubs. The outcomes were contrasted with two existing systems with demonstrate the viability of the algorithm[8].

Zhang et al (2012) proposed the situation of $\mathrm{CH}$ as an imperative factor for the system lifetime. In view of this 
perception, a non-arbitrary $\mathrm{CH}$ choice plan dependent on the idea of the focal point of mass in material science was proposed. The issue of intensity utilization in sensor information gathering in a WSN was considered. Since sensor hubs work on batteries, control effectiveness is a pivotal issue in planning the system. The geographic organization of sensor hubs is arbitrary, with a sporadic system topology. In the current bunching based conventions for the WSN, the CHs are typically chosen indiscriminately, which may result in higher power utilization and shorter system lifetime. The motivation behind proposed plot was to utilize insignificant power during the time spent information accumulation. It was demonstrated that proposed plan could set aside to half of intensity utilization [9].

Jain and Trivedi (2012) proposed a calculation for vitality proficient grouping and multi-jump directing in WSN. The sensor arrange is developed as a roundabout zone with the base station. A versatile bunch choice system which chooses the Cluster Heads (CHs) based on lingering vitality, as well as on the separation from the base station. A key purpose of the calculation is that $\mathrm{CH}$ choice is done after each round and on numerous occasions inside the equivalent round. This is done since the $\mathrm{CH}$ close to the $\mathrm{BS}$ is associated with the vast majority of the correspondences and thus it might get depleted early [10].

Wang et al (2013) proposed a Fuzzy-based framework for $\mathrm{CH}$ determination and controlling sensor speed in WSNs. In WSN, bunch arrangement and $\mathrm{CH}$ choice were basic issues. They could definitely influence the system's execution in various situations with various attributes. The 42 proposed framework was built by two Fuzzy Logic Controllers (FLC). Utilizing four info semantic parameters for assessing $\mathrm{CH}$ choice likelihood in FLC1, the yield of FLC1 was utilized and two other phonetic parameters as information parameters of FLC2 to control sensor speed. FLC2 was assessed by recreations and demonstrated that it accomplished great execution [11].

Izadi et al (2013) was proposed a sort 2 fluffy based Self-Configurable Cluster Head (SCCH) determination way to deal with consider $\mathrm{CH}$ choice model which displayed a bunch reinforcement approach. Along these lines, when bunch fizzled, the framework worked effectively. This current convention's curiosity is the capacity to deal with correspondence vulnerability, an inborn operational viewpoint in sensor systems. Results uncovered that $\mathrm{SCCH}$ performed superior to other created strategies [12].

\section{MeThODOLOGY}

In our proposed algorithm, proposer tries to overcome one of the major limitations of all varieties of LEACH. In traditional LEACH and balanced LEACH, cluster head is being changed after every round and if a node becomes cluster head for a round then it can never $\mathrm{Be} \mathrm{CH}$ for $(1 / \mathrm{P})$ rounds. where $\mathrm{p}$ is the cluster head probability, After selection of $\mathrm{CH}$ for each round whole process of cluster head creation being enacted. So it has been tried to improve the selection of cluster head and add scheme that improves the power consumption of a network.

\begin{tabular}{|c|c|c|}
\hline Sr No. & Simulation Parameter & Value Used \\
\hline 1 & Number of sensor node $(\mathrm{N})$ & 100 \\
\hline 2 & Network area $(\mathrm{M} \times \mathrm{M})$ & $100 \times 100$ \\
\hline 3 & Eelec (Transmission \& reception energy per bit) & $50 \mathrm{~nJ}$ \\
\hline 4 & $\begin{array}{l}\text { Efs (Transmit amplifier energy dissipation of free } \\
\text { space model) }\end{array}$ & $100 \mathrm{pJ} / \mathrm{bit} / \mathrm{m}^{2}$ \\
\hline 5 & $\begin{array}{l}\text { Emp (Transmit amplifier energy dissipation of two } \\
\text { model) }\end{array}$ & $0.0013 \mathrm{pJ} / \mathrm{bit} / \mathrm{m}^{4}$ \\
\hline 6 & E0(Initial energy of deployed node) & $0.25 \mathrm{~J}, 0.5 \mathrm{~J}, 1 \mathrm{~J}$ \\
\hline 7 & Eda (Data aggregation energy per bit) & $5 \mathrm{~nJ}$ \\
\hline 8 & $\mathrm{~K}$ (Number of bits in a packet) & 4000 byte \\
\hline 9 & d0 (Cross over distance) & $87 \mathrm{~m}$ \\
\hline 10 & Location of data sink & $(50,50)$ \\
\hline 11 & Popt & 0.1 \\
\hline 12 & Massage size & 4000 bits \\
\hline 13 & Number of iteration & 4000 \\
\hline
\end{tabular}

\section{Cluster Head Selection Formation Algorithm}

Step-1: Build up wireless sensor architecture. Step-2: Assign initial energy to sensor nodes.

Step-3: Sort the hubs dependent on the separation from Base station utilizing Selection Sort in expanding request. To ascertain hub separate from Base-Station the given recipe is utilized Node_distance (I)

$$
\sqrt{\left.(S(i) \cdot x d-(\sin k \cdot x)) x^{2}+(S(i) \cdot y d-(\sin k \cdot y)) x^{2}\right)}
$$

Step-4: For round=1 allocate bunch heads dependent on least separation from the base-station No of Cluster Heads for cycle $1=\left(p^{*} n\right)$. Lessening the vitality of the hubs picked as group head.

Step-5: For the next round Calculate node-degree of the chosen nominee for cluster heads

Step-6: calculate the energy of node

if $(\mathrm{S}(\mathrm{i}) \cdot \mathrm{E}=0)$ then

Dead $=\mathrm{I} / / \mathrm{i}^{\text {th }}$ node $\operatorname{dies}$

$\mathrm{n}=\mathrm{n}$-dead $/ / \mathrm{n}$ : decrease no of alive nodes

Step-7: Goto step-5

Step-8: End

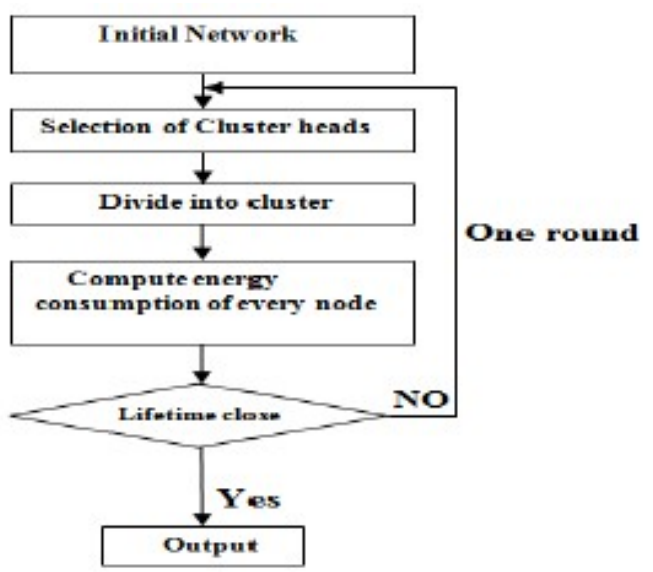

1. Setup phase:

fig 2- flow chart of algorithm

a. A group head is chosen for the simple initially round and sends the promotion (ADV) to the all the non $\mathrm{CH}$ hubs.

b. Nodes which are nearer to $\mathrm{CH}$ join the group and send the affirmation (ACK). However, the hubs which indicate powerless flag quality from 
$\mathrm{CH}$ sits tight for another ADV of various group.

c. When first round is finishes and second round begins then we check the vitality level of $\mathrm{CH}$ of the last round. In the event that the vitality level is not as much as edge then new $\mathrm{CH}$ is chosen for that round generally same $\mathrm{CH}$ is taken for next round.

d. After that $\mathrm{CH}$ makes a TDMA plan for each non $\mathrm{CH}$ hub for information transmission. In any case, if bunch head is same as that of past round then same TDMA plan is utilized with respect to the last round. After that setup stage wraps up.

2. Steady state stage:

a. Follow the TDMA calendar, and check if the correspondence between the hubs is between group or intra-bunch.

b. If the correspondence between the hubs in intrabunch at that point low power is given to them in light of the fact that less vitality expected to transmit a message inside same group. Be that as it may, a lot of vitality is expected to transmit a message outside the bunch or to the base station.

c. After that check the sensor esteem, if the sensor esteem is not as much as edge at that point sit tight for other esteem generally transmission of message begins. cluster head is selected for the very first round and sends the advertisement (ADV) to the all the non $\mathrm{CH}$ nodes.

\section{RESUlTS AND DiscuSSION}

After simulation of algorithm over MATLAB, results were taken based on some metrics like network lifetime, balance energy, count of $\mathrm{CH}$ and energy consumption and Results are discussed in following sections.

Parameters for simulation

fig-3 represent the number of nodes in simulation area for communication over the boundaries with cluster head .

\begin{tabular}{|c|c|c|c|}
\hline Energy(J/node) & protocol & $\begin{array}{c}\text { rounds } \\
\text { for first } \\
\text { node } \\
\text { dies }\end{array}$ & $\begin{array}{c}\text { rounds } \\
\text { for last } \\
\text { node } \\
\text { dies }\end{array}$ \\
\hline $0.25 \mathrm{~J}$ & $\begin{array}{c}\text { Basic } \\
\text { LEACH }\end{array}$ & 585 & 1474 \\
\cline { 2 - 4 } & $\begin{array}{c}\text { E- } \\
\text { LEACH }\end{array}$ & 1170 & 3552 \\
\hline $0.50 \mathrm{~J}$ & $\begin{array}{c}\text { Basic } \\
\text { LEACH }\end{array}$ & 1291 & 3081 \\
\cline { 2 - 4 } & $\begin{array}{c}\text { E- } \\
\text { LEACH }\end{array}$ & 1333 & 3166 \\
\hline \multirow{2}{*}{$1 \mathrm{~J}$} & $\begin{array}{c}\text { Basic } \\
\text { LEACH }\end{array}$ & 1175 & 3299 \\
& $\begin{array}{c}\text { E- } \\
\text { LEACH }\end{array}$ & 2492 & 6310 \\
\hline
\end{tabular}

fig-4 comparision table of leach protocols

\section{Result of First Dead Node}

Figure 4 demonstrates that the initial hub of Basic LEACH is expire after 585 round where as in E-LEACH convention the primary hub dead after 1170 adjusts so we can obviously say that the lifetime of first hub is increment by 2 times than ordinary LEACH.

\section{Result of Network Life}

Figure 4 demonstrates that the last hub of Basic LEACH is

\begin{tabular}{ll}
\hline Parameters & Value \\
\hline Initial energy for each sensor node & \\
Packet size & $0.5 \mathrm{~J}$ \\
Energy in idle state & $1000 \mathrm{bits}$ \\
Aggregation energy & $50 \mathrm{~nJ} / \mathrm{bit}$ \\
Inter cluster Energy when $\mathrm{d}>=\mathrm{d}_{0}$ & $5 \mathrm{~nJ} / \mathrm{bit}$ \\
\hline Inter cluster energy when $\mathrm{d}<=\mathrm{d}_{0}$ & $\mathrm{E}_{1}=10 \mathrm{pJ} / \mathrm{bit} / \mathrm{m}^{2}$ \\
\hline Intra cluster energy when $\mathrm{d}>=\mathrm{d}_{1}$ & $\mathrm{E}_{2}=0.0013 \mathrm{pJ} / \mathrm{bit} / \mathrm{m}^{2}$ \\
Intra Cluster energy when $\mathrm{d}<=\mathrm{d}_{1}$ & $\mathrm{E}_{11}=\mathrm{E}_{1} / 10$ \\
Location of $\mathrm{BS}$ & $\mathrm{E}_{22}=\mathrm{E}_{2} / 10$ \\
\hline Total number of nodes & $\mathrm{X}=50 ; \mathrm{Y}=50$ \\
Total number of rounds & 100 \\
\end{tabular}

expire after 1474 round where as in E-LEACH convention the last hub dead after 3552 adjusts so we can unmistakably say that the lifetime of last hub is increment by 3 times than Basic LEACH.

\section{CONClusion ANd Future Scope}

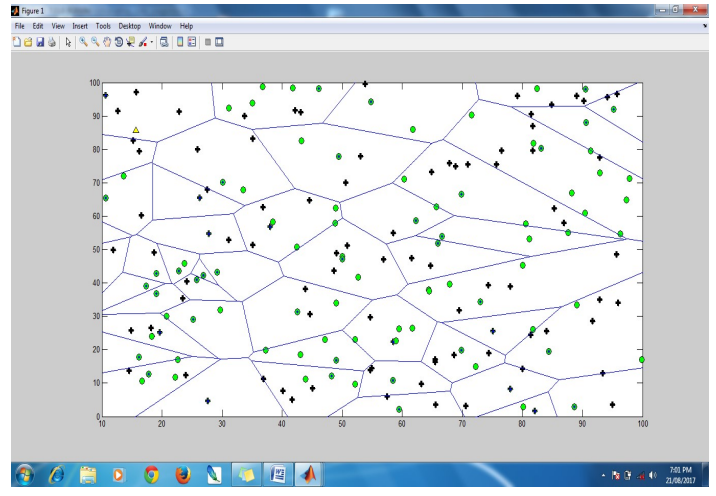

Request of vitality effective conventions are dependably in for remote sensor systems. An arrangement of vitality effective conventions (drain and its assortment) has been contemplated and discovers holes among them. in the wake of discovering holes a calculation has been proposed which is named as power-drain. The change is because of finding a typical hole i.e. determination of $\mathrm{ch}$ in each round. amid recreation of $\mathrm{e}$ filter with the current two renditions i.e. adjusted drain and fundamental filter, $\mathrm{e} \%$ and $47.19 \%$ over essential filter and adjusted filter individually. it is realized that higher the vitality utilization, most exceedingly bad the calculation is. e filter indicates critical change of 20 drain demonstrates immense change over them. the system life is ascertained by the quantity of rounds the hubs in the systems are alive. after recreation of e filter its demonstrates a change of $51.95 \%$ and $10 \%$ over essential and adjusted drain. for estimating a calculation is great or not, specialists ascertain the remaining 
vitality. on the off chance that the measure of lingering vitality is high, at that point lifetime of system is additionally high. around $47.82 \%$ and $39.13 \%$ of change is recorded over fundamental and adjusted filter. $92.4 \%$ and $92.5 \%$ change is recorded for intra bunch correspondence in e leach over other two renditions.

The proposed calculation indicates very great outcomes over essential LEACH and adjusted LEACH. Yet, regardless it doesn't demonstrate any change amid bury group information transmission when contrasted and adjusted LEACH. It might be because of too many group divisions in system while running E LEACH over it. This can be enhanced by further change in group head division calculation. This should be possible by setting a counter which chooses the quantity of groups in the system. In the event that this little change is installed in the proposed calculation, at that point it indicates great outcomes.

\section{REFERENCES}

[1] Wei Xiang, Senior Member, IEEE, Ning Wang, and Yuan Zhou, Member, IEEE "An Energy-efficient Routing Algorithm for Software-defined Wireless Sensor Networks" Citation information: DOI 10.1109/JSEN.2016.2585019, IEEE Sensors

[2] N. Lavanya* and T. Shankar " A Review on Energy-Efficient Scheduling Mechanisms in Wireless Sensor Networks" Indian Journal of Science and Technology, Vol 9(32), DOI: 10.17485/ijst/2016/v9i32/86910, August 2016.

[3] M. Chen, Y. Zhang, Y. Li, M. Hassan, and A. Alamri, "AIWAC: affective interaction through wearable computing and cloud technology," IEEE Wireless Communications, vol. 22, no. 1, pp. 20-27, Feb. 2015.
[4] Wei Wang, Qianping Wang, Wei Luo, Mengmeng Sheng, Wanrong $\mathrm{Wu}$, Li Hao, "Leach-H- An improved routing protocol for collaborative sensing networks?, Wireless Communications and Signal Processing, 2009, PP. 1:5

[5] Y. He, Y. Zhang, Y. Ji, X.S. Shen, "A new energy efficient approach by separating data collection and data report in wireless sensor networks", Proceedings of the International Conference on Communications and Mobile Computing, 2009.

[6] Min Yoon, Jaewoo Chang, "Design and Implementation of Cluster-based Routing Protocol using Message Success Rate in Sensor Networks," 2011 IEEE.

[7] Li Peng", Jian-bo xc', Wei Liang, "Inter-Cluster Route Algorithm Based on the Gateway for Wireless Sensor Networks," 2011 International Conference on Electronic \&Mechanical Engineering and Information Technology.

[8] P. Kuila, Pabitra K. Jana,"An energy balanced distributed clustering and routing algorithm for Wireless Sensor Networks" 2012 2nd IEEE International Conference on Parallel, Distributed and Grid Computing,2012.

[9] Zou, Y., Zhang, H., Jia, X.: Zone-divided and energybalanced clustering routing protocol for wireless sensor networks. In: 4th IEEE International Conference on Broadband Network and Multimedia Technology (ICBNMT), pp. 112-117 2012.

[10] Jain, N., Trivedi, P.: An adaptive sectoring and cluster head selection based multi-hop routing algorithm for WSN. In: 2012 Nirma University International Conference on Engineering (NUiCONE), pp. 1-6 2012

[11] Wang N B, Zhu H. "An energy efficient algorithm based on LEACH protocol" 2012 International Conference on Computer Science and Electronics Engineering, Hangzhou, Zhejiang, China. 2012: $339-342$.

[12] D. Izadi, J. Abawajy, and S. Ghanavati, "A new energy efficient cluster head and backup selection scheme in WSN," in Proc. IEEE 14th Int. Conf. Inf. Reuse Integr. (IRI), Aug. 2013, pp. 408-415.. 